$1-1-2005$

\title{
The factor structure of the presence questionnaire
}

Bob G. Witmer

Christian J. Jerome

University of Central Florida

Michael J. Singer

Find similar works at: https://stars.library.ucf.edu/facultybib2000 University of Central Florida Libraries http://library.ucf.edu

This Article is brought to you for free and open access by the Faculty Bibliography at STARS. It has been accepted for inclusion in Faculty Bibliography 2000s by an authorized administrator of STARS. For more information, please contactSTARS@ucf.edu.

\section{Recommended Citation}

Witmer, Bob G.; Jerome, Christian J.; and Singer, Michael J., "The factor structure of the presence questionnaire" (2005). Faculty Bibliography 2000s. 5779.

https://stars.library.ucf.edu/facultybib2000/5779

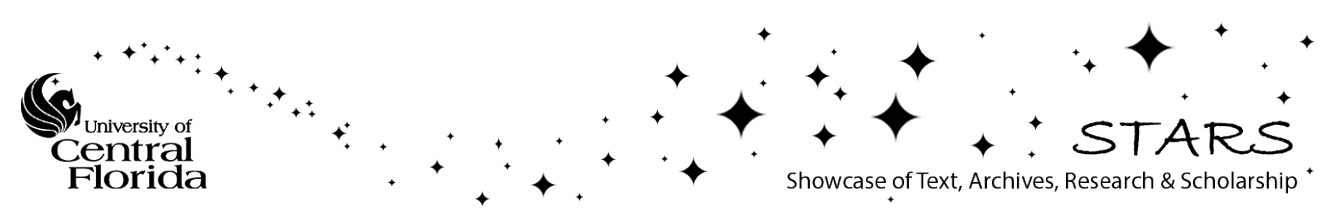




\section{Bob G. Witmer}

U.S. Army Research Institute for the Behavioral and Social Sciences Simulator Systems Research Unit Orlando FL 32826-3276 USA

\section{Christian J. Jerome}

University of Central Florida

Consortium Research Fellows

Program

\section{Michael J. Singer}

U.S. Army Research Institute for the Behavioral and Social Sciences Simulator Systems Research Unit Orlando FL 32826-3276 USA Michael.Singer@peostri.army.mil
Presence, Vol. 14, No. 3, June 2005, 298-312 (c) 2005 by the Massachusetts Institute of Technology

\section{The Factor Structure of the Presence Questionnaire}

\begin{abstract}
Constructing a valid measure of presence and discovering the factors that contribute to presence have been much sought after goals of presence researchers and at times have generated controversy among them. This paper describes the results of principal-components analyses of Presence Questionnaire (PQ) data from 325 participants following exposure to immersive virtual environments. The analyses suggest that a 4-factor model provides the best fit to our data. The factors are Involvement, Adaptation/Immersion, Sensory Fidelity, and Interface Quality. Except for the Adaptation/Immersion factor, these factors corresponded to those identified in a cluster analysis of data from an earlier version of the questionnaire. The existence of an Adaptation/Immersion factor leads us to postulate that immersion is greater for those individuals who rapidly and easily adapt to the virtual environment. The magnitudes of the correlations among the factors indicate moderately strong relationships among the 4 factors. Within these relationships, Sensory Fidelity items seem to be more closely related to Involvement, whereas Interface Quality items appear to be more closely related to Adaptation/Immersion, even though there is a moderately strong relationship between the Involvement and Adaptation/Immersion factors.
\end{abstract}

\section{Introduction}

From the first issue of this journal to the present time researchers have sought to identify the factors or variables that are responsible for the experience of presence. Early work to identify these factors was either purely conceptual or primarily empirical. The basis for early conceptual work was provided by Held and Durlach (1992), Sheridan (1992), and Zeltzer (1992) and extended by Witmer and Singer $(1994 ; 1998)$. The early theoretical work sought to define presence and related terms such as immersion, and identify factors that might add to or detract from the presence experience. For the purposes of this paper we propose the following definitions: Presence is a psychological state of "being there" mediated by an environment that engages our senses, captures our attention, and fosters our active involvement. The environment that mediates presence can be real, virtual, symbolic, or some combination thereof. The degree of presence experienced in that environment depends on the fidelity of its sensory components, the nature of the required interactions and tasks, the focus of the user's attention/concentration, and the ease with which the user adapts to the demands of the environment. It also depends on the user's previous experiences and current state. Involvement is a psychological state experienced as a consequence of focusing one's mental energy and 
attention on a coherent set of stimuli or meaningfully related activities or events. Involvement is increased by performing tasks and participating in activities that stimulate, challenge, and engage the user either cognitively, physically, or emotionally. Immersion is a psychological state characterized by perceiving oneself to be enveloped by, included in, and interacting with an environment that provides a continuous stream of stimuli and experiences. Immersion in a virtual environment (VE) is reduced by extraneous distractions and is increased by factors that facilitate direct interaction with the VE and the performance of VE task activities.

Many of the early empirical studies were conducted by Slater and his colleagues (Slater \& Usoh, 1993; Slater, Usoh, \& Steed, 1994) and Barfield and his colleagues (Barfield \& Weghorst, 1993; Barfield \& Hendrix, 1995; Hendrix \& Barfield, 1996). The primary model for these empirical investigations was to vary some aspect of the VE or its interface and assess its effect on presence via a small number of questions designed to measure presence directly. Other empirical presence research sought to explore relationships between measures of presence and performance or simulator sickness (Witmer \& Singer, 1994).

Witmer and Singer $(1994 ; 1998)$ were among the first to provide empirical data to test the notion that variables affecting presence could be classified into meaningful groups of similar items. Their cluster analysis of data from the PQ (Witmer \& Singer, 1998) produced four clusters or subscales. The Involved/Control subscale items addressed the perceived ability to control VE events, responsiveness of the VE to user-initiated actions, involvement in the visual aspects of the $\mathrm{VE}$, and overall involvement in the VE experience. The Natural subscale items measured the extent to which the interactions felt natural, the extent to which the VE was consistent with reality, and how natural the control of locomotion was through the VE. The Interface Quality items addressed whether control devices or display devices interfere or distract from task performance, and the extent to which the participants felt able to concentrate on the tasks. Two of the items, though conceptually similar, did not cluster with each other or with the other items. These items, which involve examining objects closely and examining objects from multiple view- points, were classified as Resolution items. These subscales were determined to bear similarities to the conceptual categories proposed by Witmer and Singer prior to performing the cluster analysis. Witmer and Singer (1998) noted that the results produced by the cluster analysis were preliminary and proposed that the PQ would be reexamined via a factor analysis when sufficient data became available. The generic term factor is used throughout this paper to refer to the underlying variables identified through principal-components analyses and principal-axis factoring alike.

More recently, other researchers assembled questionnaires designed to measure presence and performed factor analyses of these questionnaires (Biocca, Kim, \& Choi, 2001; Lessiter, Freeman, Keogh, \& Davidoff, 2001; Schubert, Friedmann, \& Regenbrecht, 2001). Biocca et al. asked 80 participants to either remove organs from a virtual cadaver $(n=49)$ or to remove geometric shapes $(n=25)$ using an immersive VE and then complete a 19-item presence measure. Using an exploratory principal-component factor analysis, Biocca et al. extracted 5 factors, retaining 3: Spatial Presence ( 8 items), Tactile Engagement (2 items), and Sensory Presence ( 2 items). Biocca's decision to retain three components was based on the eigenvalues, theoretical importance, and logical coherence of the items. Lessiter et al. exposed 604 participants to mediated environments representing a variety of media (3D IMAX, 2D IMAX, 2D films in general release, a short video, and a racing game). No immersive VEs were used and, except for the video game, the media involved passive viewing. The participants then completed a 63 -item presence measure developed by the authors. Lessiter et al. used the principalaxis factoring technique to extract 12 factors with eigenvalues greater than 1 , but retained only 4 . The number of items was reduced for the revised questionnaires by examining factor loadings and making reliability checks. The 4 factors were Sense of Physical Space (19 items), Engagement (13 items), Ecological Validity (5 items), and Negative Effects (6 items).

Schubert et al. (2001) in two studies asked participants $(n=246$ and $n=296)$ to complete a 75 -item questionnaire based on their last experience with any form of VE (to include desktop VE, 3D games, and immersive VE). The questionnaire included all items from 
Version 2.0 of the PQ, as well as newly developed items and selected items from other presence measures. The questionnaires were administered via the Internet or following VE exposure in Schubert's laboratory. Most of the questionnaire responses were based on $3 \mathrm{D}$ game experiences. In their first study, Schubert et al. extracted a large number of factors using principal-components analysis, but retained the following eight: Spatial Presence (14 items), Quality of Immersion (8 items), Involvement (10 items), Drama (7 items), Interface Awareness ( 7 items), Exploration of VE ( 6 items), Predictability and Interaction ( 6 items), and Realness ( 6 items). By computing scores for each factor and conducting a second-order factor analysis on the scores, Schubert et al. pared down the number of factors to three. The first factor consisted of all items from the Realness, Spatial Presence, and Involvement factors. The second factor combined items from the Interface Awareness, Predictability and Interaction, and Exploration components. The third factor included items from the Drama and Quality of Immersion components. The authors consider the first factor to be a general presence factor, while the second factor captures descriptions of the stimuli provided by the VE and its interface.

In a second study, Schubert et al. (2001) used only items from five of their eight original factors and selectively culled items from those five factors, so that 37 items from the Spatial Presence, Involvement, Exploration, Realness, and Predictability and Interaction scales remained. They used a different sample of participants, but the type and method of media exposure were very much like their first study. Seven factors with eigenvalues greater than 1 were extracted but only five were retained (the same five that were carried over from their first study). The factors were Spatial Presence (14 items), Exploration (8 items), Realness (3 items), Predictability and Interaction ( 2 items), and Involvement (10 items).

What conclusions may be drawn from these various factor analyses of presence measures? How do the results of these analyses differ and how are they alike? What is the true structure of presence? The most noticeable similarity among the three factor analyses is that a spatial component is common to all. Each analysis also isolates an involvement or engagement component. There is a component in each that addresses how much the mediated environment is like the real world, although this was not immediately apparent based on the different labels (Sensory Presence, Realness, and Ecological Validity) assigned to that component by the different researchers. However, there were also some major differences in the three studies. The number and types of factors vary across studies. Measures with more items typically produce a larger number of factors (i.e., more factors with eigenvalues exceeding 1.0). Factors and items are frequently retained or discarded based on criteria that vary from one researcher to the next and can at times involve subjective judgments or a preference for particular item types. This suggests that the factors reveal more about the structure of the particular presence measure and the researcher's theoretical preferences than about the underlying structure of the presence concept. This does not mean that factor analyses of presence measures cannot provide valuable insights about the presence construct, but it would not be prudent to interpret factors obtained from these or similar studies as representing the true and complete structure of the presence concept. However, the research described above is notable in that each of these researchers attempted to provide reliable, valid, and comprehensive measures of presence that included a number of different types of items. This is in contrast to some previous efforts that used only a small number of relatively homogeneous, face-valid items to measure presence (Slater, Usoh, \& Steed, 1994; Slater, Linakus, Usoh, \& Kooper, 1996).

The purpose of the present paper is to describe the results of a principal-components factor analysis of the Witmer and Singer Presence Questionnaire (PQ-Version 3.0) and identify the basic factors underlying that particular measure of presence. Because the PQ has been widely distributed and used as a measure of presence in a variety of settings (e.g., Renaud, Rouleau, Granger, Barsetti, \& Bouchard, 2002; Stanney, Kingdon, Graeber, \& Kennedy, 2002; Vora et al., 2002), it is important to understand its basic structure in terms of underlying factors. A second purpose is to develop additional insight that might provide further information regarding the presence construct. Previous work done 
by Schubert et al. (2001), Biocca et al. (2001), and Lessiter et al. (2001) was very helpful in planning our analysis and instrumental in generating ideas for this investigation. However, there are notable differences between the current work and these previous studies. The current work is based on presence data collected immediately following exposure to "immersive virtual environments." Of the three factor-analytic studies, only Biocca et al. obtained their presence measures following exposure exclusively to immersive VE. Lessiter et al. purposely exposed participants to a variety of media forms, while Schubert's data were weighted heavily toward exposure to 3D computer games. Also, Schubert's data were not always collected immediately after media exposure- a condition that could alter reported presence. In the current study, like Biocca et al., we used immersive VE exclusively, but we surveyed more participants and exposed them to a wider range of VE interfaces and activities than did Biocca and his colleagues.

\section{Background}

Witmer and Singer (1998) investigated the structure of Version 2.0 of the Presence Questionnaire (32 items) by conducting a cluster analysis of PQ data. Only those PQ items that contributed to the reliability of the scale (19 items) were included in the analysis. PQ haptics and auditory items were removed from the scale because including them reduced scale reliability as measured by Cronbach's alpha. Using PQ data from four experiments $(N=152)$, the cluster analysis identified the following PQ subscales: Involved/Control (11 items), Natural (3 items), Interface Quality (3 items), and Resolution ( 2 items). The 19 reliable items formed the core for Version 3.0 of the Presence Questionnaire. In addition to the 19 items, Version 3.0 included 3 auditory items and 2 haptic items from Version 2.0 and 8 new or revised items for a total of 32 items. The total PQ score for Version 3.0 was based only on the 19 core items with demonstrated reliability (renumbered in Version 3.0 as items $1-4,6-10,14-16$, and $18-24)$. The remaining "research items" were retained to determine their utility as potential additions to the presence scale. Research items include auditory items (5, 11, \& 12), haptic items ( $13 \& 17)$, and the new/revised items (items 25-32). The item stems included in the PQ Version 3.0 are shown in Table 1 .

\section{Method}

\section{I Sample}

The sample for the current analyses was made up of 201 men and 124 women ( 325 total), who had participated in one of seven experiments. Except for 34 infantry soldiers participating in one experiment, the participants were university students. The participants ranged in age from 18 to 47 years $(M=22.79, S D=$ 4.99). None of these participants was included in the research reported in Witmer and Singer (1998). Researchers at the Simulator Systems Research Unit of the Army Research Institute for the Behavioral and Social Sciences in Orlando, Florida collected the data during experiments in our VE labs at the University of Central Florida Institute for Simulation and Training or at facilities at Fort Benning.

\subsection{Study Procedures}

In each experiment the participants completed Version 3.0 of the PQ immediately after performing tasks in a virtual environment. Among the tasks were direction and distance estimation, learning building layouts, terrain familiarization, interior search tasks, and simulated combat in urban environments. All experiments involved immersive virtual environments, using either the VR4 or V8 HMD, or the Fakespace BOOM as the viewing device. The locomotion interface was either a treadmill, a joystick, or a motion-tracking system for walking in place. The VEs ranged from a simple hallway to a complex building or detailed outside terrain. All of the VEs incorporated some type of audio stimuli, but only one of the seven experiments incorporated directional sound. None of the VEs used touchsensitive gloves or force-feedback devices. Haptic stimulation was provided via the locomotion interfaces and contact with hand-controller devices. There were also indirect virtual haptic effects (e.g., doors opening when approached, or forward motion impeded by simulated 
Table I. Presence Questionnaire Item Stems (Version 3.0)

1. How much were you able to control events?

2. How responsive was the environment to actions that you initiated (or performed)?

3. How natural did your interactions with the environment seem?

4. How much did the visual aspects of the environment involve you?

5. How much did the auditory aspects of the environment involve you?

6. How natural was the mechanism which controlled movement through the environment?

7. How compelling was your sense of objects moving through space?

8. How much did your experiences in the virtual environment seem consistent with your real world experiences?

9. Were you able to anticipate what would happen next in response to the actions that you performed?

10. How completely were you able to actively survey or search the environment using vision?

11. How well could you identify sounds?

12. How well could you localize sounds?

13. How well could you actively survey or search the virtual environment using touch?

14. How compelling was your sense of moving around inside the virtual environment?

15. How closely were you able to examine objects?

16. How well could you examine objects from multiple viewpoints?

17. How well could you move or manipulate objects in the virtual environment?

18. How involved were you in the virtual environment experience?

19. How much delay did you experience between your actions and expected outcomes?

20. How quickly did you adjust to the virtual environment experience?

21. How proficient in moving and interacting with the virtual environment did you feel at the end of the experience?

22. How much did the visual display quality interfere or distract you from performing assigned tasks or required activities?

23. How much did the control devices interfere with the performance of assigned tasks or with other activities?

24. How well could you concentrate on the assigned tasks or required activities rather than on the mechanisms used to perform those tasks or activities?

25. How completely were your senses engaged in this experience?

26. To what extent did events occurring outside the virtual environment distract from your experience in the virtual environment?

27. Overall, how much did you focus on using the display and control devices instead of the virtual experience and experimental tasks?

28. Were you involved in the experimental task to the extent that you lost track of time?

29. How easy was it to identify objects through physical interaction, like touching an object, walking over a surface, or bumping into a wall or object?

30. Were there moments during the virtual environment experience when you felt completely focused on the task or environment?

31. How easily did you adjust to the control devices used to interact with the virtual environment?

32. Was the information provided through different senses in the virtual environment (e.g., vision, hearing, touch) consistent? 
solid objects). The PQ data from the seven experiments were aggregated and analyzed using the Statistical Package for the Social Sciences (SPSS). A series of analyses examined the structure of the PQ. Principal-components analysis with direct oblimin rotation was the method used in all analyses. The term factor is used throughout this paper to refer to the components identified in these analyses. Items were assigned to a factor if they loaded highest on that factor, regardless of multiple factor loadings.

\subsection{Study I: Comparing Cluster Analysis and Principal-Components Analysis Results}

3.3.I Purpose and Method. The purpose of the first study was to compare the factors identified by principal-components analysis of the current data with the results from the earlier cluster analysis of PQ data (Witmer \& Singer, 1998). The core 19 PQ items from Version 3.0 were subjected to a principalcomponents analysis using the new data $(N=325)$. Factors with eigenvalues greater than 1 were retained. A Kaiser-Meyer Olkin (KMO) measure of 0.91 indicated high sampling adequacy and excellent preconditions for the principal-components factor analyses.

3.3.2 Results. Factor 1 consisted of PQ items 1 , $2,3,4,6,7,8,14$, and 18 (see Table 1). Items 1 and 2 were control items, while Items $4,7,14$, and 18 were involvement items. These items correspond almost exactly with the items comprising the Involved/Control subscale from the earlier cluster analysis. Three additional items $(3,6, \& 8)$ that had formed a separate subscale labeled Natural in that cluster analysis were also part of Factor 1 in the present analysis. An interface that is natural to the user immediately facilitates the user's ability to control activities in the environment, increasing the user's involvement. Factor 1, henceforth called Involvement, accounted for $37.4 \%$ of the variance.

Factor 2 consisted of 2 items (Items $22 \& 23$ ). These items, accounting for $9.4 \%$ of the variance, measure the perceived quality of the VE interface and the extent to which it does not interfere with activities in the VE.
These 2 items plus Item 24 had formed the Interface Quality subscale in the earlier cluster analysis. We retain that subscale label for Factor 2.

Factor 3 consisted of 6 items (Items 9, 10, 19, 20, $21, \& 24)$. In the prior cluster analysis (Witmer \& Singer, 1998), the first 5 items were part of a 12 -item Involved/Control subscale, while the last item was a member of the Interface Quality subscale. These items address the perceived proficiency of interacting with and operating in the VE and how quickly the user adjusted to the VE experience. In one sense, better VE task performance and quickly adapting to the new environment suggests that the user is more immersed in that environment. Participants who adjust quickly and readily to the $\mathrm{VE}$ and its interfaces are more likely to feel immersed in the VE. Therefore, we named this third factor $A d$ aptation/Immersion. More will be said about this later. This factor accounts for $6.3 \%$ of the variance in this analysis.

Factor 4 consisted of only two items (Items $15 \&$ 16). These two conceptually similar items, previously referred to as PQ Resolution, were expected to be linked. These items address the degree to which the VE configuration permits active search or survey of and/or examination of the objects in the VE using vision. We have renamed this factor Visual Fidelity because it represents the fidelity of the visual sense in the VE. The Visual Fidelity factor accounts for $5.7 \%$ of the variance.

3.3.3 Discussion. The principal-components analysis of the 19 core items for PQ Version 3.0 both supports and adds to our previous cluster analysis results. Factor 1 combines elements from two previously separate subscales (Involved/Control and Natural), while Factors 2 and 4 correspond to subscales previously identified in the cluster analysis. The newly identified subscale, Adaptation/Immersion, is an important addition, providing empirical evidence of a separate immersion factor. The previous cluster analysis of the PQ failed to reveal an immersion subscale, though Witmer and Singer (1998) had argued that involvement and immersion were major components of presence. 


\subsection{Study 2: Expanding the Principal- Components Analysis to Include PQ Research Items}

3.4.I Purpose and Method. The purpose of Study 2 was to determine the suitability of the 13 PQ Version 3.0 research items that are not currently scored for inclusion in the PQ scale, and include suitable items in an expanded principal-components analysis. Items that are suitable should not reduce the PQ scale reliability when added to the original 19-item PQ scale. First, the 13 research items and 19 original items were merged to form a 32-item scale, and scale reliability was computed using Cronbach's alpha. Then items were removed from the 32 -item PQ scale one at a time with replacement. After the removal of each new item, the iterative application of Cronbach's alpha assessed PQ scale reliability. Three research items $(26,27, \& 28)$ were dropped from subsequent analyses because they tended to reduce PQ scale reliability when included; the remaining items comprised a reliable 29-item PQ scale. Principal-components analysis with direct oblimin rotation of the data from the 29 remaining items produced an interpretable factor structure. Direct oblimin rotation was used to allow for expected correlations among the factors.

3.4.2 Results. Cronbach's alpha for the 29 -item scale was $0.91(N=325)$. Six factors were extracted using a minimum eigenvalue of 1.0 as the criteria. $\mathrm{Cu}^{-}$ mulatively the six-factor solution accounted for $60.1 \%$ of the variance.

Factor 1, Involvement (Items 1, 2, 3, 4, 6, 7, 8, 10, $14, \& 18$ ), accounted for $31.9 \%$ of the variance. Except for Item 10, the items were identical to Involvement factor items identified in Study 1.

Factor 2 encompassed 3 of the original 19 items (Items $5,11, \& 12$ ) and accounted for $8.8 \%$ of the variance. The first item is a direct measure of the participant's involvement with the audio aspects of the VE, while the other items measured the perceived fidelity of the auditory aspects. Therefore this factor was labeled Auditory Fidelity.

Factor 3 encompassed three items from the Adaptation/Immersion factor identified in Study 1 (Items 20,
$21, \& 24)$, and added four new items $(25,30,31, \&$ 32 ). Item 21 relates to the user's proficiency in interacting with the $\mathrm{VE}$, while Item 24 addresses concentration on the VE task as opposed to concentration on VE interface devices. Item 25 asks how completely the VE experience engaged the senses, and was added to address sensory immersion. Item 32 measures whether the information experienced through different sensory modalities seemed consistent. Higher sensory consistency would appear to be related to higher levels of sensory immersion. Item 30 measures the participant's sense of being completely focused on the VE and the task being performed in that environment. Note that this item also measures immersion, but in a more cognitive sense. Items 20 and 31 measure how well participants felt that they adjusted or adapted to the VE experience and the VE control interfaces. Both of these items also ask participants about their interactions with the VE. Items 9, 10 , and 19, which had loaded highly on this factor in Study 1, migrated to other factors (see below). The Adaptation/Immersion factor accounted for $6.5 \%$ of the variance.

Factor 4, Interface Quality (Items 22 \& 23), accounted for $5 \%$ of the variance. Items 22 and 23 , the core items for this factor, measure the quality of the visual and control interfaces, respectively.

Factor 5 (Items $9 \& 19$ ) accounted for $4 \%$ of the variance. This factor is new and was not separately identified in previous analyses. Item 9 had been in the Adaptation/Immersion subscale (see Study 1), but migrated to the new Factor 5 in this study. Item 9 measures the extent to which the environment reacts to user-initiated actions as expected, and Item 19 measures perceived delay between user actions and environment reactions. In the real world there is seldom any perceptible delay between user action and environment reactions. Therefore any noticeable delay in the VE would not be consistent with the participant's expectations. Hence we named this factor Consistent with Expectations.

Factor 6 is composed of three touch-related research items (Items 13, 17, \& 29) and two vision-related factors (Items 15 \& 16). This factor, labeled Haptic/Visual Fidelity, indicates the degree to which the VE configuration permits users to examine objects visually, or to search for, examine, and manipulate objects using 
their sense of touch. Haptic/Visual Fidelity factor accounted for $3.8 \%$ of the variance.

3.4.3 Discussion. The six-factor solution of the expanded PQ included all of the factors identified in Study 1 for the core PQ, with some minor rearrangements. Factor 1, Involvement, Factor 3, Adaptation/ Immersion, and Factor 4, Interface Quality, were found in both studies. Factors 2 and 6 in this study include sensory fidelity items, some of which also appeared in Study 1 under the Visual Fidelity Factor. Factor 5 in this study, Consistent with Expectations, was not extracted in Study 1. Factor 5 indicates the extent that the VE reacts to participants' actions as expected, and reflects participants' attempts to integrate sensory input in accordance with their expectations. These expectations are likely based on the user's previous experience, primarily with the real world.

Although the six-factor solution accounts for $60 \%$ of the variance and initially appears to be an optimal solution, inspection of the scree plot coupled with the small number of items in some factors suggests otherwise. The scree plot helps the analyst to determine the optimum number of factors to retain by ordering the eigenvalues of the factors from highest to lowest. This allows the analyst to visually separate a subset of factors with relatively high eigenvalues that show a smooth decline along the curve's slope from a subset of factors with relatively low eigenvalues that represent the factorial debris at the bottom of the curve. Examination of the scree plot suggests that the three-factor, four-factor, or five-factor solutions might fit the data equally well, if not better, than the six-factor solution. In the present analysis, the optimal number of factors was not obvious from the inspection of the simple scree plot (represented by circles in Figure 1), suggesting the need for a different technique.

\subsection{Study 3: Determining the "Correct" Number of Factors-The Four-Factor Solution}

3.5.I Purpose and Method. The purpose of this study was to determine the correct number of factors to retain to represent the structure of the PQ. As stated previously, inspecting the scree plot did not clearly show where the cutoff point fell (three, four, five, or six factors). Some researchers simply include all factors above an eigenvalue of 1 . This method is simple and straightforward; however, it may overestimate the number of factors. Also, it can be argued that this may inflate the number of factors due to sampling error. An extant method is commonly used to subtract out the component in the latent roots that can be attributed to sampling error (Horn, 1965). Horn suggests a procedure that involves generating the same sized data matrix from a normally distributed universe of random numbers, then reducing the data using the same factor-analysis methods used in the real-data analysis. Finally, the scree plots for the real and random data sets are superimposed and the point where the two lines cross is considered the cutoff for the number of factors in the data (for a detailed explanation of these methods, refer to Horn, 1965).

For this study, a 32 by 325 data matrix was generated using Microsoft Excel. Each variable (column) was generated independently for each subject (row) from a normally distributed universe of random numbers. These data were then analyzed using principal-components analysis - the same factor-analytic methods that were used to analyze the real presence data.

3.5.2 Results. The results of the comparison between the scree plots for the randomly generated data and the real presence data are shown in Figure 1. As can be seen from Figure 1, the lines cross at the fourth factor. Thus, the four-factor solution will be adopted as the better of the competing solutions. The four-factor model accounted for $52.2 \%$ of the variance. Factor 1 , Involvement, accounted for $31.9 \%$ of the variance and included Items 1, 2, 3, 4, 6, 7, 8, 10, 14, 17, 18, and 29. Factor 2, Sensory Fidelity, accounted for $8.8 \%$ of the variance, and included Items $5,11,12,13,15$, and 16. Factor 3, Adaptation/Immersion, accounted for $6.5 \%$ of the variance, and included Items $9,20,21,24$, $25,30,31$, and 32. Factor 4, Interface Quality (Items $19,22, \& 23)$, accounted for $5.0 \%$ of the variance. Internal consistency reliability coefficients were computed for each of the four factors using Cronbach's alpha. Except for the Interface Quality Factor $(a l p h a=.57)$, 


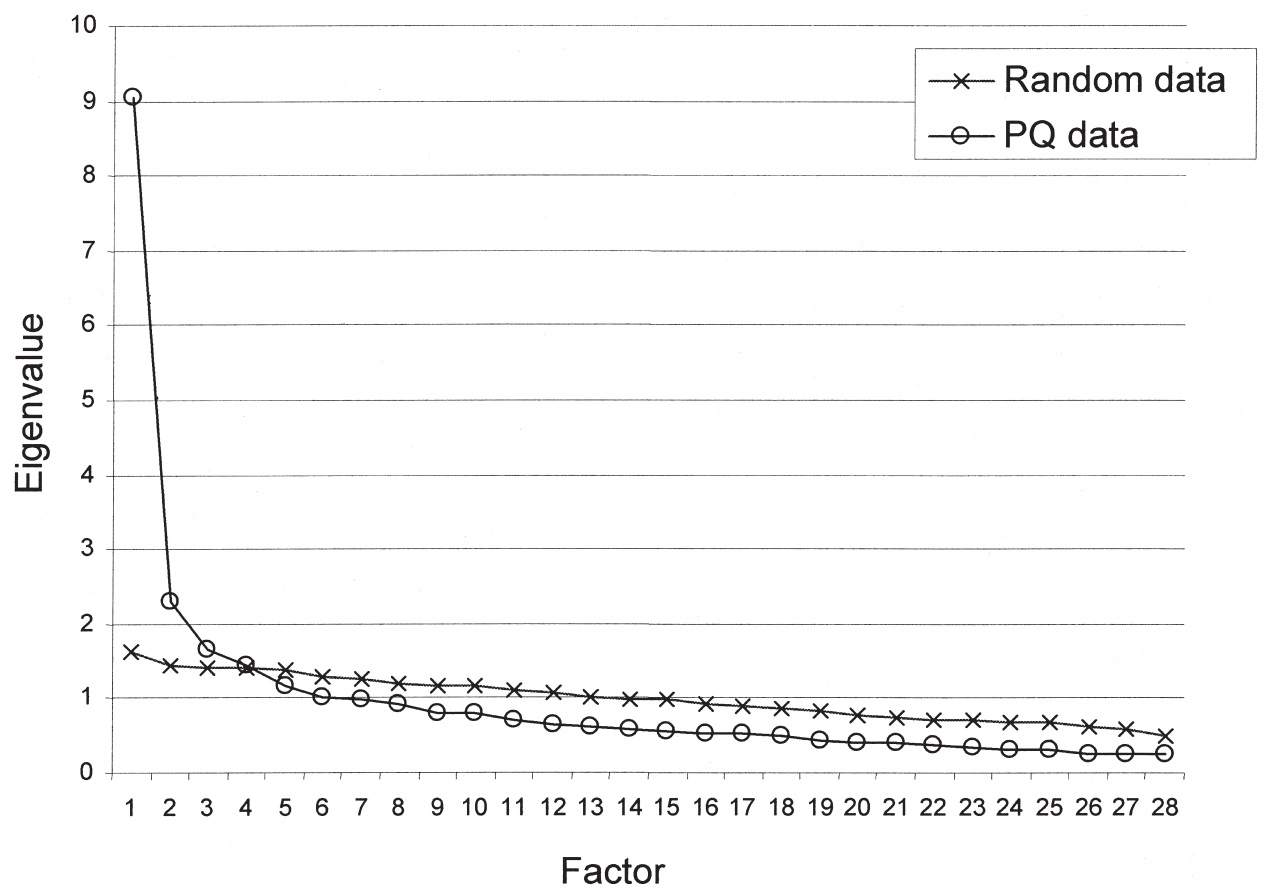

Figure I. Scree plots showing random data vs. PQ data.

which consists of only three items, the reliability coefficients were quite good: Involvement (alpha $=.89$ ), Sensory Fidelity (alpha $=.84$ ), and Adaptation/Immersion $(a l p h a=.80)$. No attempt was made to increase the reliability of the individual subscales by removing items that reduce subscale reliability, because that procedure would likely reduce the overall reliability of the PQ.

Factor loadings for each of the PQ items are presented in Table 2. All items load at least .30 on one or more factors. Roughly one third of the items in the four-factor model showed clear patterns of cross loading on more than one factor. A decision was made to retain items having high loadings on more than a single factor. We felt it was more important to retain items that contributed substantial variance to the total PQ score than to form conceptually pure factors. Despite multiple loadings, items were assigned to one, and only one, factor, based on their highest loading. This assignment of each item to only one factor simplifies the computation of PQ totals and factor subscale scores. For each factor, marker variables are indicated by item numbers enclosed in parentheses. We used Bedford's (1997) criteria to identify marker variables. Marker variables are items that loaded .30 or higher on a factor and loaded at least .20 higher on that factor than any cross loading. Marker variables are those variables that uniquely define a factor. Each of the four factors had a minimum of three marker variables.

The direct oblimin rotation procedure used in the analysis of the four-factor model produces correlated factors. The correlations among the factors are shown in Figure 2. There are moderate correlations between Involvement and Adaptation/Immersion, and between Involvement and Sensory Fidelity. Smaller but still significant correlations were observed between Interface Quality and Adaptation/Immersion, and between Sensory Fidelity and Adaptation/Immersion. While Sensory Fidelity is related to both Involvement and Adaptation/Immersion, it is more highly correlated with Involvement. Interface Quality, on the other hand, seems to be related to Adaptation/Immersion, but its correlation with Involvement is very low and not significant. 
Table 2. Factor Loadings (Pattern Matrix) for 29-Item Presence Questionnaire 4-Factor Model with Direct Oblimin Rotation

\begin{tabular}{|c|c|c|c|c|}
\hline \multirow{2}{*}{$\begin{array}{l}\text { PQ Item \# } \\
\text { (from Table } 1 \text { ) }\end{array}$} & \multicolumn{4}{|c|}{ 4-Factor model factor loadings } \\
\hline & Involvement & Sensory fidelity & Adaptation/immersion & Interface quality \\
\hline 1 & $.724(1)$ & .070 & .121 & -.014 \\
\hline 2 & $.669(2)$ & .119 & .108 & -.006 \\
\hline 3 & $.807(3)$ & .065 & .046 & .086 \\
\hline 4 & $.630(4)$ & -.089 & .141 & -.111 \\
\hline 5 & .024 & $-.814(5)$ & .038 & .011 \\
\hline 6 & $.766(6)$ & .052 & -.085 & .182 \\
\hline 7 & $.634(7)$ & -.095 & .115 & -.069 \\
\hline 8 & $.768(8)$ & .051 & .052 & .061 \\
\hline 9 & .211 & -.107 & .354 & .104 \\
\hline 10 & .384 & -.073 & .364 & .055 \\
\hline 11 & -.175 & $-.940(11)$ & .076 & .045 \\
\hline 12 & -.115 & $-.926(12)$ & .005 & .051 \\
\hline 13 & .410 & -.428 & -.323 & .025 \\
\hline 14 & $.614(14)$ & -.045 & .301 & -.165 \\
\hline 15 & .292 & -.452 & .198 & -.209 \\
\hline 16 & .342 & -.359 & .246 & -.249 \\
\hline 17 & .479 & -.334 & -.304 & .034 \\
\hline 18 & .428 & -.161 & .419 & -.220 \\
\hline 19 & -.034 & -.030 & .064 & $.557(19)$ \\
\hline 20 & .213 & -.006 & .427 & .285 \\
\hline 21 & .261 & .002 & $.572(21)$ & .107 \\
\hline 22 & -.006 & -.150 & .114 & $.655(22)$ \\
\hline 23 & .140 & .087 & -.065 & $.736(23)$ \\
\hline 24 & .034 & .116 & $.669(24)$ & .063 \\
\hline 25 & .155 & -.128 & $.573(25)$ & -.124 \\
\hline 29 & .354 & -.322 & .031 & -.019 \\
\hline 30 & -.090 & -.089 & $.712(30)$ & -.101 \\
\hline 31 & -.007 & -.050 & $.563(31)$ & .269 \\
\hline 32 & -.050 & -.374 & .449 & .099 \\
\hline
\end{tabular}

3.5.3 Discussion. We used Horn's (1965) method to determine the correct number of factors to retain. This method is an objective procedure that has been shown to provide more accurate estimates of the number of factors to be retained than simply retaining all factors with eigenvalues greater than 1 (Zwick \& Velicer, 1986). It is not subject to the experimenter bias that can occur when factors are retained based solely on factor interpretability or theoretical considerations. The four-factor model used in Study 3 simplified the factor structure of the 29-item PQ to four basic elements that we labeled Involvement, Adaptation/Immersion, Sensory Fidelity, and Interface Quality. The Sensory Fidelity factor includes visual, auditory, and haptic items. The Adaptation/Immersion factor in the four-factor model included Item 9 (ability to anticipate system re- 


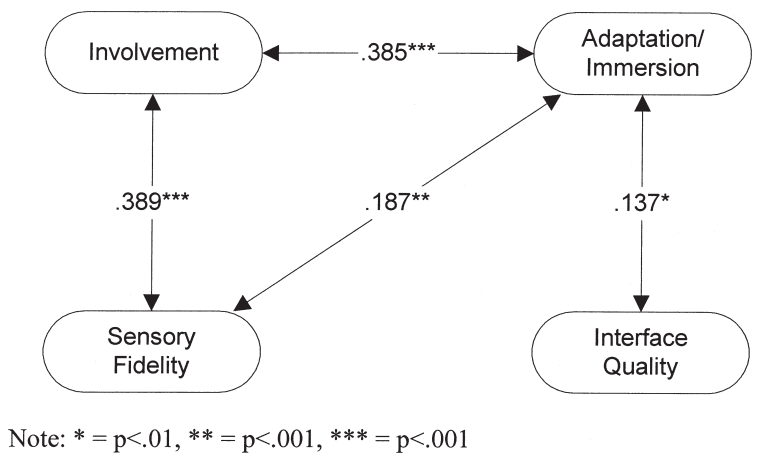

Figure 2. $P Q$ between-factor correlations.

sponse to user actions) plus all of the Adaptation/Immersion items previously identified in Study 2. The Involvement factor included all of the involvement items from Study 2 plus Item 17, a control item involving object manipulation, and Item 29 (a haptic item that would seem better included under Sensory Fidelity). Note that Item 29 loads almost as high on the Sensory Fidelity factor as it does on the Involvement factor (see Table 1). The Interface Quality factor includes the same two items identified in the previous studies plus Item 19. Item 19 measures perceived delay between actions and outcomes and hence could be affected by the quality of the VE interface.

The magnitude of the correlations among the factors indicates that although the analysis of the four-factor model provides clearly interpretable, statistically separated factors, the factors share common variance. The dominant factor in the 29-item PQ was Involvement. It accounted for $31.9 \%$ of the PQ Variance. Both the Sensory Fidelity factors and the Adaptation/Immersion factors shared a significant proportion of their variance (15\%) with Involvement. It is interesting to consider Item 18, a conceptually pure involvement item; Item 18 loaded almost as high on Adaptation/Immersion as it did on the Involvement factor. This illustrates the significant interdependence between Involvement and Adaptation/Immersion. As shown in Figure 2, Adaptation/Immersion shared significant variance with the Interface Quality factor (2\%) and the Sensory Fidelity factor $(3.5 \%)$. The fact that factors share variance does not reduce their value as determinants of presence. All of these factors are needed to provide a comprehensive multidimensional measure of presence.

\section{General Discussion}

The four factors identified for the 29-item PQ in Study 3 are remarkably similar to the factors identified for the 19-item PQ from Study 1 and replicate four of the six factors extracted in Study 2. Table 3 shows the items associated with each factor for each of the factoranalytic solutions and also how the factors map onto each other from one model solution to the next. For each model the factors are not arranged in numerical order, but are placed in the table so that the factors for each model and the items within the factors can be tracked from left to right for each subsequent analysis. Marker variables, items that loaded .30 or higher on a factor and loaded at least .20 higher on that factor than on any other factor, are shown in bold font in the table.

\section{I Involvement, Adaptation/ Immersion, and Sensory Fidelity Factors}

In Witmer and Singer (1998), involvement was defined as a psychological state experienced as a consequence of focusing one's mental energy and attention on a coherent set of stimuli or meaningfully related activities or events. Immersion was defined as a psychological state characterized by perceiving oneself to be enveloped by, included in, and interacting with an environment that provides a continuous stream of stimuli and experiences. Factor analyses of the PQ suggests these separate but related factors are both measured by the PQ. By conducting the analyses we have gained additional insight regarding these factors. Involvement is clearly the most dominant dimension measured by the PQ. It appeared in our earliest analysis and continues to appear in all subsequent analyses, and the items composing it changed little from one study to the next. The significant relationship between sensory fidelity and involvement determined in Study 3 suggests that sensory fidelity might influence involvement indirectly through its effects on the user's energy and attention. High sen- 
Table 3. Factor Analytic Model Solutions for 19-Item and 29-Item Versions of the PQ

\begin{tabular}{|c|c|c|}
\hline 19-item PQ (from Vs. 2.0) & 29-item PQ (from Vs. 3.0) & \\
\hline 4-Factor model (Study l) & 6-Factor model (Study 2) & 4-Factor model (Study 3) \\
\hline $\begin{array}{l}\text { 1. Involvement }(1,2,3,4 \text {, } \\
\quad 6,7,8,14,18)\end{array}$ & $\begin{array}{l}\text { 1. Involvement }(1,2,3,4,6,7,8,10 \text {, } \\
14,18)\end{array}$ & $\begin{array}{l}\text { 1. Involvement }(1,2,3,4,6,7,8,10 \text {, } \\
14,17,18,29)\end{array}$ \\
\hline 4. Visual fidelity $(15,16)$ & $\begin{array}{l}\text { 2. Audio fidelity }(5,11,12) \\
\text { 6. Haptic/visual fidelity }(13,15,16 \text {, } \\
17,29)\end{array}$ & 2. Sensory fidelity $(5,11,12,13,15,16)$ \\
\hline $\begin{array}{l}\text { 3. Adaptation/immersion } \\
(9,10,19,20,21,24)\end{array}$ & $\begin{array}{l}\text { 3. Adaptation/immersion }(20,21,24 \text {, } \\
25,30,31,32) \\
\text { 5. Consistent with expectations }(9,19)\end{array}$ & $\begin{array}{l}\text { 3. Adaptation/immersion }(9,20, \mathbf{2 1}, \mathbf{2 4} \text {, } \\
\mathbf{2 5}, \mathbf{3 0}, \mathbf{3 1}, \mathbf{3 2})\end{array}$ \\
\hline 2. Interface quality $(22,23)$ & 4. Interface quality $(22,23)$ & 4. Interface quality $(\mathbf{1 9}, \mathbf{2 2}, \mathbf{2 3})$ \\
\hline
\end{tabular}

Note: For the 4-factor model, marker variables are shown in bold typeface.

sory fidelity media are more likely to capture the user's attention, supporting a greater level of involvement. Based on the relative magnitude of the correlations among the factors, low sensory fidelity might be expected to have a greater impact on involvement than it does on immersion. ${ }^{1}$ This likely occurs because poor sensory fidelity tends to distract and divert one's energy and attention from the required tasks and activities and focus it on processing the incoming information. The sense of immersion or inclusion might be less affected by poor sensory fidelity. Some participants readily adapt to low-fidelity VEs and become immersed. Hence, a low sensory fidelity environment may still produce considerable immersion. Books are a prime example of low sensory fidelity media that may produce high levels of immersion.

\subsection{Adaptation/Immersion and Interface Quality Factors}

The Adaptation/Immersion factor seems to be less stable, with items shifting in and out of that factor from one study to the next. However, three items (20, $21, \& 24)$ loaded most highly on this factor in all three

1. Although larger correlations may suggest stronger ties between factors and lead to the formulation of causal hypotheses, it is important to remember that correlations do not test a causal hypothesis. Testing causal hypothesis requires other methods (e.g., controlled experiments, structural equation modeling). studies. Item 20 asked participants how quickly they adjusted to the VE and Item 21 asked about the proficiency in interacting with the VE. Item 24 asked how well they concentrated on the VE activities. Three additional items $(25,30, \& 31)$ loaded highest on this factor in two of the studies. Item 25 asked participants how completely their senses were engaged in the VE experience; Item 30 asked how completely they focused on the VE, while Item 31 also asked participants how well they adjusted. Taken together, the items included in this factor suggest that the degree of immersion may depend in part on how completely participants can concentrate on VE activities, enabling them to adjust quickly to their new surroundings and acquire proficiency in interacting with the VE.

The concentration aspect of immersion may explain the link between involvement and immersion. Concentration seems to facilitate both involvement and immersion by directing and focusing attention on the relevant VE activities. However, immersion seems to be more closely related to the participant's ability to adapt to the VE than does involvement. The user may become involved either before adapting to the VE or even if little adaptation occurs. Immersion, on the other hand, seems to require adapting to the VE or other media. Interface quality, which correlates significantly with the Adaptation/Immersion factor, should affect both how fast par- 
ticipants adapt to the VE and how well they perform in the VE. A poor interface is likely to produce performance deficits and increase the time it takes to adapt to the VE. An individual's ability to rapidly adapt to the VE may be a key component of immersion in virtual environments. Development of task proficiency within the VE may be another key indicator of the degree of immersion.

These aspects of immersion typically have not been associated with the immersion concept. For example, Slater and Wilbur (1997) describe immersion as being indicative of the extent to which computer-driven displays are capable of delivering an inclusive, extensive, vivid, and surrounding illusion of reality. They also list other dimensions of immersion, most of which have a spatial component. We do not deny that there is a strong spatial (or perceived emplacement) component to immersion. Indeed our definition of immersion (Witmer \& Singer, 1998) also emphasizes these spatial aspects. But to better understand what underlies the immersive experience, we as researchers must be open to including other variables that may influence this experience. Slater and Wilbur, for example, include story line or plot as a dimension of immersion, which is a departure from its typical spatial aspects. It is important not to discard variables simply because they do not conform to our preconceived notions of immersion and presence.

\section{Summary and Conclusions}

\section{I The Structure of the Presence Questionnaire}

Through a series of factor analyses and reliability analyses, a new 29-item version of the Presence Questionnaire Scale has been developed and the factor structure of this scale has been revealed. The PQ includes the following four factors or subscales: Factor 1 , Involvement (Items 1, 2, 3, 4, 6, 7, 8, 10, 14, 17, 18, \& 29 from Table 1); Factor 2, Sensory Fidelity (Items 5, 11, $12,13,15, \& 16$ from Table 1); Factor 3, Adaptation/ Immersion (Items 9, 20, 21, 24, 25, 30, 31, \& 32 from Table 1); and Factor 4, Interface Quality (Items 19, 22, \& 23 from Table 1$)$.

Taken together, these subscales provide a robust, reliable measure of presence that can be used with confi- dence by researchers. The PQ attempts to cover as many aspects of presence as possible in order to provide a multifaceted presence measure. The PQ asks participants to report their perceptions of the experiences provided by the VE sensory and control interfaces, their involvement in the VE task, the nature and quality of their interactions with the VE, and how quickly they adjusted to the VE experience. All of these aspects influence presence, and should be measured. Not to include as many aspects of presence as possible in a presence survey is a missed opportunity. As a multifaceted, multidimensional measure of presence, the PQ does not represent an attempt to produce a theoretically pure measure in which all of the survey items must explicitly resemble the presence concept as represented by its definition. There are only so many ways one can ask the basic question, "How strong was your feeling of being there or of being in a real place?" A measurement approach that relies on a small number of relatively homogeneous items for representing the phenomenon of presence is unlikely to provide adequate coverage of the determinants of presence and is likely to be statistically unreliable. Instead, the PQ seeks to provide a comprehensive measure of presence that captures the multitude of variables that influence the presence experience. The PQ asks participants to report their perceptions of the target environment and their interactions with that environment, not just their subjective feelings of presence. As such the PQ provides a broad and balanced measure of the presence experience.

\subsection{Relationship of Current Findings to Previous Findings}

The factor structure of the 29-item PQ was identified using a single set of data. With a different data set the item loadings and factors could change somewhat. However, the fact that the current subscales identified using factor-analytic techniques correspond roughly to the subscales identified in the cluster analysis of a different data set speaks for the relative stability of three of the four factors. It is also important to note that our most robust subscale, Involvement (or engagement), had also been isolated in factor analyses by other researchers (Lessiter et al., 2001; Schubert et al., 2001). 
Similarly, the sensory-presence factor identified by Biocca, et al. (2001) may be related to our Sensory Fidelity factor. The items composing Biocca's sensorypresence factor differ from our sensory-fidelity items in that the former asked participants how much their sensory experiences in the VE were like their real-world experiences, while our sensory-fidelity items asked participants how well they were able to sense different aspects of the VE (without asking for a direct comparison to the real world). Based on the strength of the relationship between our adaptation and immersion items, we believe that our Adaptation/Immersion subscale is related to spatial factors identified in previous factor analyses (Biocca et al., 2001; Lessiter et al., 2001, \& Schubert et al., 2001). The items composing these spatial factors correspond closely with our definition of immersion. Our Interface Quality factor was not identified by other researchers, partly because the items that they selected and/or retained for their analyses did not ask respondents to assess the quality of the VE interfaces.

\subsection{New Immersion Items}

The only new subscale identified in the current analyses, Adaptation/Immersion, was identified in the principal-components analysis of the 19-item PQ and bolstered by the inclusion of four new Adaptation/Immersion items in the 29-item PQ. Adding a small number of items that directly address the spatial component of immersion might further increase the face validity of the Adaptation/Immersion subscale and its relation to presence. Although a small number of PQ items seem to address sensory and cognitive immersion (e.g., Items $25,30, \& 32$ ), inspection of Table 1 suggests that the purely spatial component of immersion is not "directly" measured by the current PQ Version 3.0 items. Four potential immersion items with spatial components are listed below. These items were selected based on our definition of immersion (see Section 4.1) and are similar to items included by other authors under the terms "Spatial Presence" (Biocca et al., 2001; Schubert et al., 2001) or "Sense of Physical Space" (Lessiter et al., 2001). Items A 3 and A4 are variants of items used by Slater and his colleagues (Slater et al., 1994). We pro- pose to add these four items as experimental items to our next version of the presence questionnaire:

Al. To what extent did you feel completely surrounded by and enveloped by the virtual environment?

A2. As you moved through the virtual environment and interacted with it, did you feel like you were inside the virtual environment, affecting or being affected by objects and events in that environment?

A3. How much did your experience in the virtual environment seem like you were in a real place, able to directly sense and interact with the environment?

A4. In the virtual environment, how strong was your sense of "being there"?

Adding these items to our current Adaptation/Immersion items that measure adaptation to the $\mathrm{VE}$ and perceived performance proficiency in the VE should result in a robust Adaptation/Immersion subscale that encompasses a spatial component, as well as the sensory and cognitive aspects of immersion. It will be interesting to determine if these four spatial items tend to cluster with other items in our Adaptation/ Immersion subscale as expected, cluster with items from other subscales, or form a separate spatial presence factor.

\subsection{Need for Additional Research}

Presence is a relatively young concept and our understanding of it remains incomplete and subject to change. Principal-components factor analysis is just one tool that may help to better understand the factors underlying the concept. Although less well known than factor analysis, structural equation modeling is another tool that may aid in discovering the relationships among the variables underlying presence, and perhaps also the relationship between presence and other variables such as simulator sickness, individual immersive tendencies, or performance (Jerome \& Witmer, 2002). Measures of presence, including the $\mathrm{PQ}$, must be tested and retested if we are to have confidence in their validity. It is hoped that the analyses presented in this paper in some way help in achieving a better understanding of the concept of presence and the PQ as a measure of presence. 


\section{References}

Barfield, W., \& Hendrix, C. (1995). The effect of update rate on the sense of presence within virtual environments. Virtual Reality: Research, Development, and Application, I(1).

Barfield, W., \& Weghorst, S. (1993). The sense of presence within virtual environments: A conceptual framework. Proceedings of the Fifth International Conference on HumanComputer Interaction, 2, 699-704.

Bedford, A. (1997). On Clarke-Watson's tripartite model of anxiety and depression. Psychological Reports, 80, 125-126.

Biocca, F., Kim, J., \& Choi, Y. (2001). Visual touch in virtual environments: An exploratory study of presence, multimodal interfaces, and cross-modal sensory illusions. Presence: Teleoperators and Virtual Environments, 10(3), 247-265.

Held, R., \& Durlach, N. (1992). Telepresence. Presence: Teleoperators and Virtual Environments, 1(1), 109-112.

Hendrix, C., \& Barfield, W. (1996). Presence within virtual environments as a function of visual display parameters. Presence: Teleoperators and Virtual Environments, 5(3), 274-289.

Horn, J. L. (1965). A rationale and test for the number of factors in factor analysis. Psychometrika, 30, 179-185.

Jerome, C. J., \& Witmer, B. G. (2002, October). Immersive tendency, feeling of presence, and simulator sickness: Formulation of a causal model. Proceedings of the Forty-sixth Annual Meeting of the Human Factors and Ergonomics Society, 2197-2201.

Lessiter, J., Freeman, J., Keogh, E., \& Davidoff, J. (2001). A cross-media presence questionnaire: The ITC sense of presence inventory. Presence: Teleoperators and Virtual Environments, 10(3), 282-297.

Renaud, P., Rouleau, J., Granger, L., Barsetti, I., \& Bouchard, S. (2002). Measuring sexual preferences in virtual reality: A pilot study. Cyberpsychology and Behavior, 5(1), $1-9$.

Schubert, T., Friedman, F., \& Regenbrecht, H. (2001). The experience of presence: Factor analytic insights. Presence: Teleoperators and Virtual Environments, 10(3), 266-281.
Sheridan, T. B. (1992). Musings on telepresence and virtual presence. Presence: Teleoperators and Virtual Environments, l(1), 120-125.

Slater, M., Linakis, V., Usoh, M., \& Kooper, R. (1996, July). Immersion, presence, and performance in virtual environments: An experiment using tri-dimensional chess. Paper presented at the 1996 Virtual Reality and Software and Technology Conference, Hong Kong.

Slater, M., \& Usoh, M. (1993). Representation systems, perceptual position, and presence in immersive virtual environments. Presence: Teleoperators and Virtual Environments, 2(3), 221-233.

Slater, M., Usoh, M., \& Steed, A. (1994). Depth of presence in virtual environments. Presence: Teleoperators and Virtual Environments, 3(2), 130-144.

Slater, M., \& Wilbur, S. (1997). A framework for immersive virtual environments (FIVE): Speculations on the role of presence in virtual environments. Presence: Teleoperators and Virtual Environments, 6(6), 603-616.

Stanney, K. M., Kingdon, K. S., Graeber, D., \& Kennedy, R. S. (2002). Human performance in immersive virtual environments: Effects of exposure duration, user control, and scene complexity. Human Performance, 15(4), 339-366.

Vora, J., Nair, S., Gramopadye, A. K., Duchowski, A. T., Melloy, B. J., \& Kanke, B. (2002). Using virtual reality technology for aircraft visual inspection training: Presence and comparative studies. Applied Ergonomics, 33(6), 559-570.

Witmer, B. G., \& Singer, M. J. (1994). Measuring immersion in virtual environments. (ARI Technical Report 1014). Alexandria, VA: U. S. Army Research Institute for the Behavioral and Social Sciences.

Witmer, B. G., \& Singer, M. J. (1998). Measuring presence in virtual environments: A presence questionnaire. Presence: Teleoperators and Virtual Environments, 7(3), 225-240.

Zeltzer, D. (1992). Autonomy, interaction and presence. Presence: Teleoperators and Virtual Environments, 1(1), 127-132.

Zwick, W. R., \& Velicer, W. F. (1986). Comparison of five rules for determining the number of components to retain. Psychological Bulletin, 99, 432-442. 